\title{
A Model for the Metastability Delay of Sequential Elements*
}

\author{
Thomas Polzer ${ }^{\dagger}$ and Andreas Steininger* \\ Embedded Computing Systems Group, \\ Institute of Computer Engineering, \\ Technische Universität Wien, Treitlstrasse 3, A-1040 Vienna, Austria \\ ${ }^{\dagger}$ tpolzer@ecs.tuwien.ac.at \\ tsteininger@ecs.tuwien.ac.at
}

Received 2 October 2016

Accepted 20 January 2017

Published 9 March 2017

\begin{abstract}
It is well known that every sequential element may become metastable when provided with marginal inputs, such as input transitions occurring too close or input voltage not reaching a defined HI or LO level. In this case the sequential element requires extra time to decide which digital output level to finally present, which is perceived as an output delay. The amount of this delay depends on how close the element's state is to the balance point, at which the delay may, theoretically, become infinite. While metastability can be safely avoided within a closed timing domain, it cannot be completely ruled out at timing domain boundaries. Therefore it is important to quantify its effect. Traditionally this is done by means of a "mean time between upsets" (MTBU) which gives the expected interval between two metastable upsets. The latter is defined as the event of latching the still undecided output of one sequential element by a subsequent one. However, such a definition only makes sense in a time-safe environment like a synchronous design. In this paper we will extend the scope to so-called value-safe environments, in which a sequential element can safely finalize its decision, since the subsequent one waits for completion before capturing its output. Here metastability is not a matter of "failure" but a performance issue, and hence characterization by MTBU is not intuitive. Therefore we will put the focus on the delay aspect and derive a suitable model. This model extends existing approaches by also including the area of very weak metastability and thus providing complete coverage. We will show its validity through comparison with transistor-level simulation results for the most popular sequential elements in different implementations, point out its relation to the traditional MTBU model parameters, namely $\tau$ and $T_{0}$, and show how to use it for calculating the performance penalty in a value-safe environment.
\end{abstract}

Keywords: Comparison of metastability behaviors; metastability characterization; asynchronous logic; Muller C-element; RS-latch; mutex; model.

\footnotetext{
*This paper was recommended by Regional Editor Zoran Stamenkovic.

$\$$ Corresponding author.

This is an Open Access article published by World Scientific Publishing Company. It is distributed under the terms of the Creative Commons Attribution 4.0 (CC-BY) License. Further distribution of this work is permitted, provided the original work is properly cited.
} 


\section{Introduction}

Whenever a mapping from a continuous space to a discrete one needs to be performed, metastability enters the scene. In digital logic this becomes relevant (a) in the voltage domain, when mapping the analog voltage level on the signal rail to a logic $H I$ or $L O$ level; and (b) in the time domain when a decision needs to be made on the order of transitions originating from unrelated time domains. The threat of metastability always shows up at the borderline cases, and its result is, in the very frequent case of a sequential element that is supposed to change its state according to the result of the decision, a delay of that decision. In fact, Marino has proven that this decision time cannot be bounded. ${ }^{1}$

Different types of basic circuit elements exist that evaluate different kinds of decision. The sequential elements used in digital design, like the D-flip-flop, the RSlatch, etc., all operate in the time domain, i.e., in one way or another their state depends on the temporal relation of their input transitions. As a result they become metastable when these transitions occur too close to each other.

It is well understood under which conditions metastability can occur and how to deal with it. In synchronous designs specific synchronizer circuits are employed to align non-synchronous inputs to the time grid in a reliable way. There are also proven ways of calculating the reliability of such synchronizers, in terms of the mean time between upsets (MTBU) based on the relevant characteristics of the sequential element in question.

In the handshake-driven asynchronous circuits different basic elements, like the mutual exclusion element (mutex), are used to handle signals originating outside the handshake domain. However, here it is not so clear how to characterize their metastability-related properties, and to the best of our knowledge no proven standard approaches exist.

In this paper we will show that the MTBU approach fundamentally does not fit the needs of asynchronous elements, and elaborate a suitable alternative. To this end we will, in Sec. 2, revisit the model underlying the synchronous characterization approach that is widely used. We will adapt it accordingly in Sec. 3, first considering the area of relatively deep metastability, and then extending the model towards weak metastability. Next, in Sec. 4 we will illustrate how to fit the model parameters to results from measurement or simulation. We will show that a good fit can be achieved for all popular types of sequential elements and different implementations thereof, and we will apply our proposed approach to study the differences. We will also leverage the closed analytic expression of the complete delay graph provided by our model to calculate the performance penalty incurred by occasional metastability resolution in a system with elastic timing. Finally we will conclude the paper with Sec. 5 . 


\section{Background}

\subsection{Metastability model for the D-latch}

Veendrick has derived a model for the metastable behavior of a D-latch. ${ }^{2}$ In accordance with the typical implementation he decomposes the storage cell into two crosscoupled inverters. Each of these inverters, in turn, is modeled as a linear voltage amplifier with a first-order low-pass behavior. For the resulting dynamic system the differential equations are derived. They show that, when left on its own under initial conditions close to (actually $V_{\Delta}$ apart from) the equilibrium state between $H I$ and $L O$ outputs, i.e., the metastable balance point, the storage cell's output voltage follows an exponential function towards the stable $H I$ or $L O$ state. The time constant $\tau$ of this function is determined by the model amplifiers' gain $A$ and time constant $R C$ :

$$
V_{\text {out }}(t)=V_{\Delta} \cdot e^{\frac{t}{\tau}}
$$

By transformation of Eq. (1) it can be shown that the time $t_{\text {res }}$ it takes for a metastable state to resolve towards a given threshold $V_{\text {th }}$ (applied by the input of the subsequent stage to judge $V_{\text {out }}$ as being $H I$ or $L O$ ) depends on the initial difference voltage according to

$$
t_{\mathrm{res}}\left(V_{\Delta}\right)=\tau \cdot \ln \left(\frac{V_{\mathrm{th}}}{V_{\Delta}}\right) .
$$

This shows that in essence any resolution time can be experienced - with a $V_{\Delta}$ of zero one even gets infinite $t_{\text {res}}$, which is also confirmed by Marino's theoretical proofs. $^{1}$

In a next step Veendrick considers a periodic clock signal with frequency $f_{\text {clk }}$ connected to the enable input of the latch, as well as an input with average transition rate $\lambda_{\text {dat }}$ at the data input. From these assumptions a uniform distribution of $V_{\Delta}$ over the undefined voltage range between $\mathrm{HI}$ and $\mathrm{LO}$ can be derived. The underlying approach here is to establish a linear relation between the voltage domain $\left(V_{\Delta}\right)$ and the time domain (temporal displacement $\Delta T$ between active clock edge and the considered data transition) by assuming a linear slope $S$ of the input transition:

$$
V_{\Delta}=S \cdot \Delta T
$$

In a synchronous system the latch output has a given resolution time $\hat{t}_{\text {res }}$ available (typically determined by the clock period minus the applicable path delays and setup time of the next stage), so based on Eq. (2) and the knowledge about the uniform distribution of $V_{\Delta}$ the average rate of upsets can be calculated, with "upset" being defined as a case where the actual resolution time $t_{\text {res }}$ exceeds the available one $\hat{t}_{\text {res }}$. The inverse of this rate is defined as mean time between upsets and can be 
calculated as

$$
\mathrm{MTBU}=\frac{1}{f_{\mathrm{clk}} \cdot \lambda_{\mathrm{dat}} \cdot T_{0}} \cdot e^{\frac{\hat{t}_{\mathrm{res}}}{\tau}},
$$

with $T_{0}$ being defined as

$$
T_{0}=\frac{V_{\mathrm{th}, \mathrm{HI}}-V_{\mathrm{th}, \mathrm{LO}}}{S},
$$

i.e., the quotient of size of the "forbidden range" between clean $H I$ and $L O$ states and the input slope.

Equation (4) is fundamental for the design of synchronizers. It is also used to experimentally determine the metastability characteristics $\tau$ and $T_{0}$ of a given latch or flip-flop. To this end the MTBU is measured for several different resolution times $\hat{t}_{\text {res }}$. It follows from Eq. (4) that in a semilogarithmic plot the curve log(MTBU) over

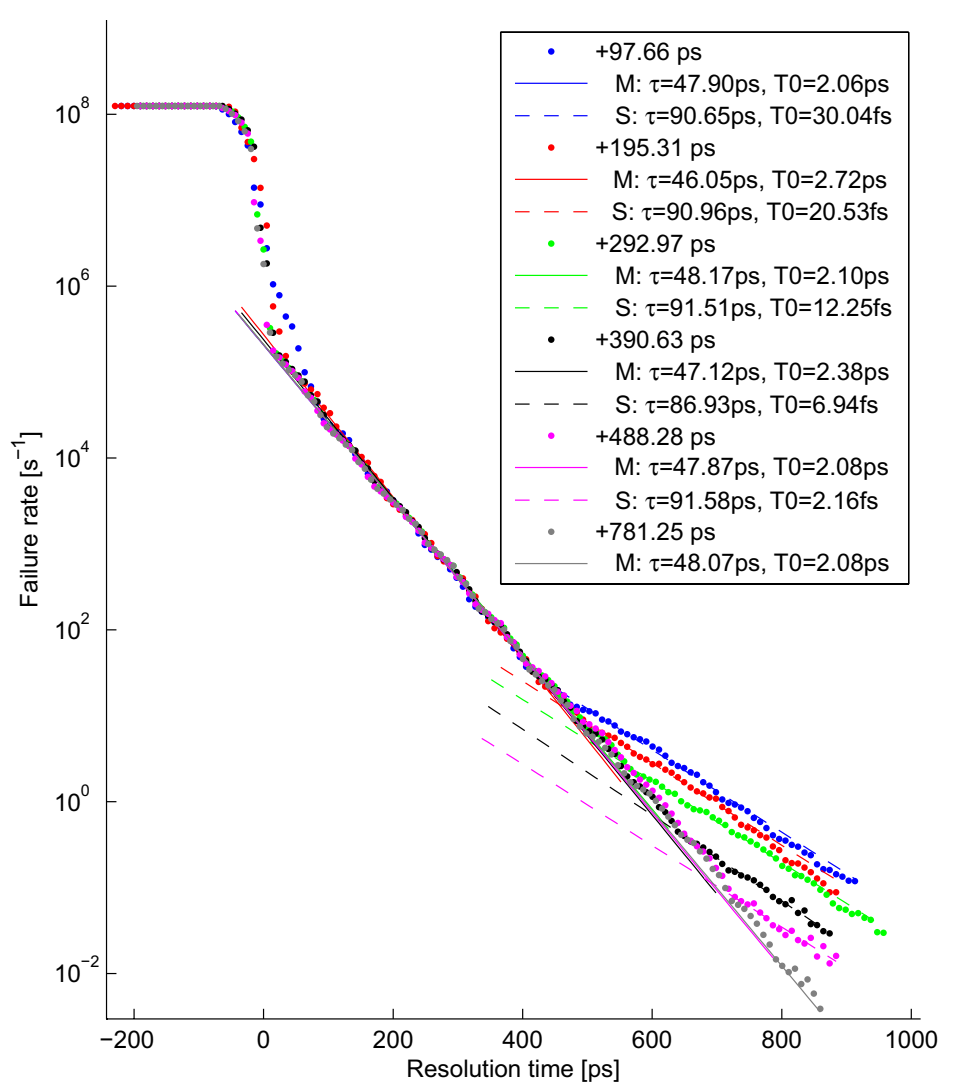

Fig. 1. The D-flip-flop MTBU measurement result (example for a $90 \mathrm{~nm}$ FPGA). 
$\hat{t}_{\text {res }}$ is a straight line with slope being determined by $\tau$ and offset by $T_{0}$. Figure 1 shows an example.

Alternatively, $\tau$ could be determined from the relevant transistor and circuit parameters using appropriate AC models, but this is more intricate and hence rarely used..$^{3,4}$

\subsection{Sequential elements for synchronous environments}

By far the most popular sequential element in synchronous designs is the $D$-flip-flop. Its function is to output the logic state present at its data input at an instant defined by the active (typically rising) transition on the clock input that is typically connected to the global synchronous clock.

A different, time-centered, view on the D-flip-flop's function is to decide whether a given transition on the data has occurred before or after the closest active clock edge. The D-flip-flop becomes metastable if these transitions coincide, i.e., when some intermediate voltage level that occurs during the data transition is sampled. In a closed synchronous design where all timing conditions are respected, this will not occur. However, at timing domain boundaries metastability becomes a problem.

Although metastability is clearly an undesired effect for a D-flip-flop, the metastable state is unproblematic, as long as it resolves fast enough to allow an output response within the available resolution time. Therefore, fast resolution, as expressed by a small $\tau$, is the central metastability-related property of a D-flip-flop.

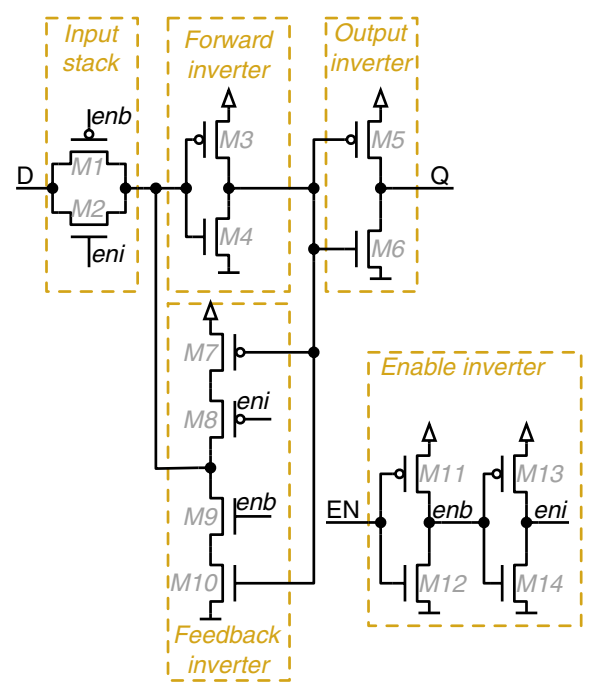

(a) D-latch.

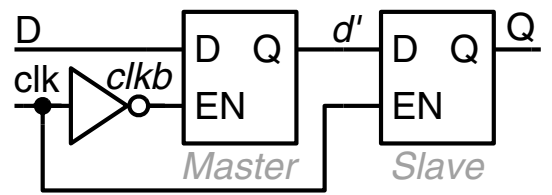

(b) Master-slave arrangement.

Fig. 2. The D-flip-flop circuit. 
The standard implementation of the D-flip-flop is based on two successive D-latch stages with the first one being driven by an inverted clock, as shown in Fig. 2. Each of these stages contains a storage loop that is formed by two cross-coupled inverters. Measurement of the relevant metastability parameters is performed by late transition detectors, as illustrated in context with Fig. 1.

\subsection{Sequential elements for asynchronous environments}

Probably the most heavily used sequential element in asynchronous circuits is the Muller C-element (MCE). Its function is to output the logic state of its (typically, but not necessarily, two) inputs if they match, and to keep the last valid output state otherwise. Its usual application is to form an AND for transitions, i.e., to issue an output transition only after having seen the same transition at all its inputs (implementation of the "wait for all" paradigm in asynchronous designs).

The MCE becomes metastable when an output transition is triggered but then withdrawn before being completed, which can happen for coincident contradicting input transitions and for short input pulses. ${ }^{5}$ Again, in a closed asynchronous design that respects the indication principle such conditions will never occur. ${ }^{6}$

For MCE implementation different circuits have been proposed, each of which coming with different benefits and drawbacks. Figure 3 displays the most popular ones; for a more in-depth discussion see literature. ${ }^{7}$

All implementations essentially consist of an input stack and an inverter loop. The differences are just in details, as the conventional implementation gates the feedback path when both inputs match, and the van Berkel implementation combines the feedback and input stacks together. Due to this structure, the same models

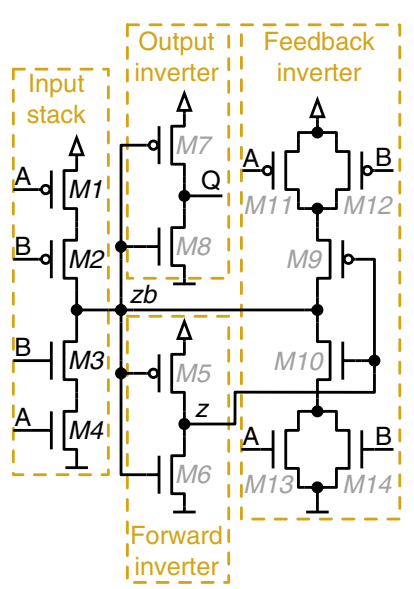

(a) Conventional.

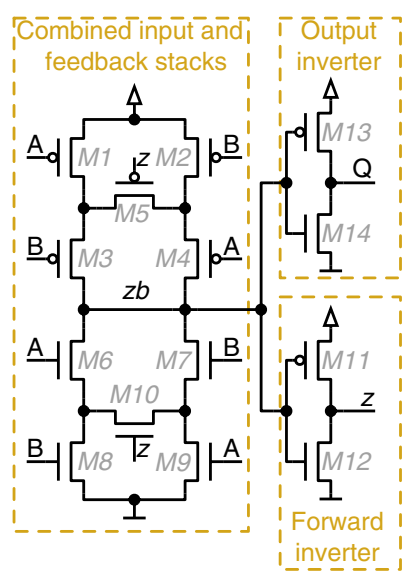

(b) van Berkel.

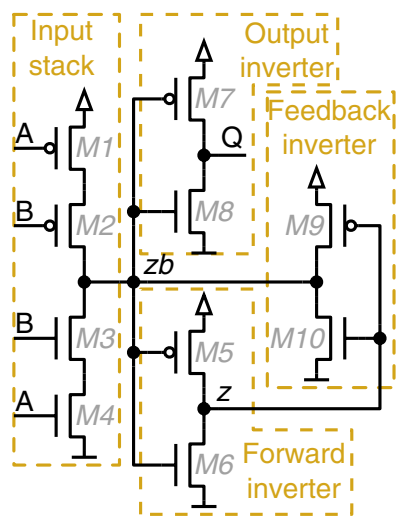

(c) Weak feedback (FB).

Fig. 3. The Muller C-element circuits. 
as for the D-latch are valid and the same basic metastability behavior has to be assumed.

The measurement of the metastability parameters by means of the late transition detector is not straightforward, as it is not a priori clear which of the input transitions the delay measurement needs to be related to (in retrospective, the transition that arrives later is the relevant one). However, as we have shown previously, the late transition detection circuit can be carefully adapted to this purpose. ${ }^{8,5}$

Another fundamental sequential function block is the $R S$-latch. Its function is to set the output to $H I$ when its set input is activated, and to set it to $L O$ when its reset input is activated. Activating both inputs is undesired, as it leads to a contradiction. It becomes metastable when (a) a marginal input pulse (insufficient length or amplitude) is applied or (b) both inputs are activated and then concurrently deactivated.

A typical application of the RS-latch is the implementation of a function with noncomplementary set and reset functions. ${ }^{a}$ The implementation of the RS-latch is typically based on two cross-coupled NAND gates, as shown in Fig. 4(a). As long as the set and reset inputs are both at $H I$, they can be abstracted away, which reduces the circuit to the well-known pair of cross-coupled inverters. There is no dedicated input stack; the inputs rather directly interfere with the storage loop.

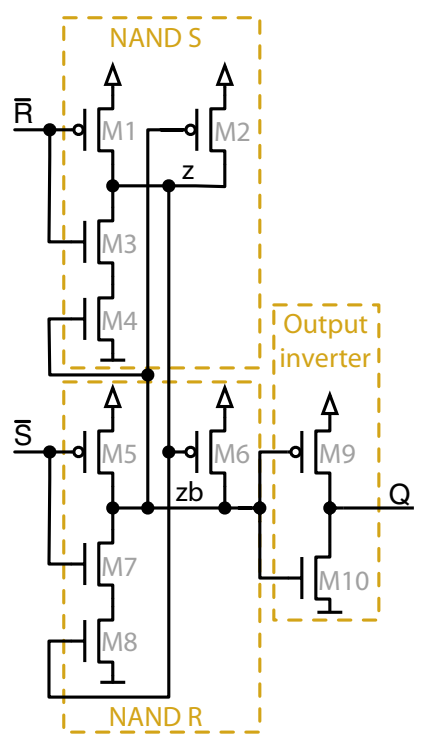

(a) RS-latch.

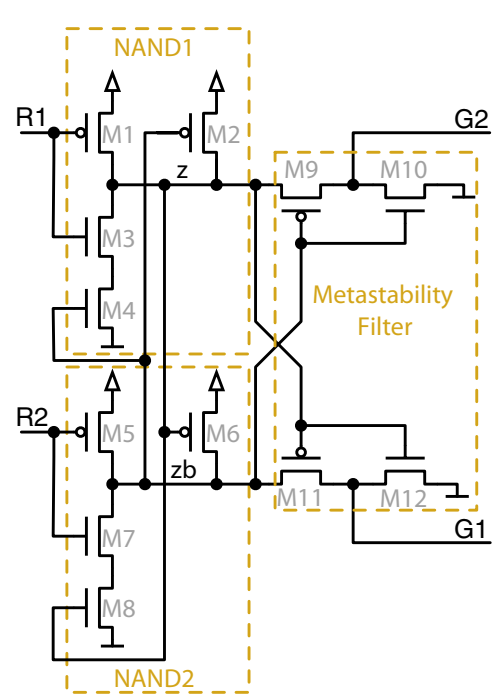

(b) Mutex.

Fig. 4. The RS-latch and mutex circuits.

\footnotetext{
${ }^{\text {a }}$ In the case of complementary set and reset functions we have a combinational element.
} 
Like in the case of MCE the experimental assessment of the metastability parameters by means of a late transition detection circuit requires specific enhancements to account for the fact that no clear reference transition is available for measuring the output delay, as is the case for the D-flip-flop.

The mutual exclusion element is another fundamental building block in asynchronous designs. Its purpose is to decide which of its (normally two) request inputs arrived earlier and to activate its associated grant output. Such a functionality is required when managing the access of several (unsynchronized) clients to a shared resource. The mutex may become metastable when the requests arrive concurrently, in which case the decision becomes arbitrary. Here it does not matter how long the mutex takes to decide (this is just a matter of performance, not of reliability), however, no more than one grant output may be activated at any time. To enforce this, an appropriate metastability filter needs to be employed that keeps all grant outputs from being activated during the metastable state.

Figure 4(b) shows the most popular implementation, as proposed by Seitz. ${ }^{9}$ It is an RS-latch with a metastability filter attached to its outputs.

With the RS-latch forming the heart of the mutex implementation, the parameter measurement can be accomplished in the same way as described above for the former.

\subsection{Time-safe versus value-safe}

It is important to note that in a synchronous design the points in time at which an output must be available, are strictly dictated by the clock, and hence the available resolution time is strictly limited. This is called a time-safe approach, and the price for demanding a result in limited time is the risk of sampling an intermediate value, i.e., experiencing a metastable upset. It has been formally proven that metastability cannot be completely avoided in such a setting, albeit it can be made arbitrarily improbable by increasing the allowed resolution time.

In contrast to that, for a mutex, for example, it is of utmost importance to never activate both grant outputs at the same time. The price for enforcing this (by means of the metastability filter) is that the actual resolution time is theoretically unlimited. This is called a "value-safe" approach. It is underlying essentially all asynchronous design styles, especially the (quasi)delay-insensitive one.

Note that metastability occurs in both, synchronous and asynchronous systems. In both cases it can be avoided for signals within the considered timing domain (clock or handshake) by appropriate design. At the timing domain boundaries, however, metastability problems generally emerge (also in the case of external faults). Here the decision for one of these approaches or the other makes a fundamental difference in how the metastable state is understood: In a time-safe environment metastability is associated with the risk of experiencing a metastable upset, hence the characterization by means of the MTBU. In a value-safe environment, in contrast, metastability results in a delayed (but still correct) decision and ultimately becomes a 
performance issue - here a characterization by means of an MTBU does not make sense, or is at best not intuitive. Therefore we are proposing an alternative in this paper.

\section{The Delay Graph}

\subsection{Model adaption}

Recall from Veendrick's model as presented in Sec. 2.1 that the natural behavior of a bistable element is the metastability resolution described by the exponential rise of the output voltage (Eq. (1)) as well as the delayed transition perceived by the subsequent stage, as expressed in Eq. (2). In contrast, the notion of an "upset" and the subsequent calculation of an MTBU is based on the assumption of a synchronous environment: Only if a limit $\hat{t}_{\text {res }}$ for the resolution time is imposed by the environment, the actual resolution time $t_{\text {res }}$ can be considered excessive. There is, however, an important class of systems that are based on an elastic timing in the sense that activities are not driven by a rigid global clock but rather by a local handshake that establishes a closed-loop timing control which adapts to delays, such as the one caused by metastability resolution. A typical example are (quasi)delay-insensitive asynchronous circuits. ${ }^{6}$ In such environments it does not make sense to use MTBU for characterization. Still, however, these systems typically comprise bistable elements, such as a Muller C-element, an RS-latch or a mutex. It is well understood that, just like the D-latch, these elements also exhibit a delay when resolving a metastable state, but to the best of our knowledge there is no established way of characterizing their metastable behavior in a meaningful way. For these elements the actually relevant property is the output delay they incur when resolving from a given initial $V_{\Delta}$. This delay, albeit not critical for the reliable function, will cause a performance penalty and is hence a property that needs consideration. This is especially true when comparing different implementation options.

In Sec. 2.3 we have seen that all the bistable elements found in asynchronous circuits essentially contain the same type of storage element formed by cross-coupled inverters, or at least can be reduced to such a structure while being in hold mode. Consequently we can still apply Veendrick's modeling approach, however, only up to the point where an externally imposed resolution time enters the scene. More specifically, Eqs. (1) and (2) are still valid, and we can use the latter to display a graph of $t_{\text {res }}$ over $V_{\Delta}$. In practice, $V_{\Delta}$ will be dependent on some type of timing relation $\Delta T$ between the input signals, just like in the case of Veendrick's D-latch. In the case of mutex (as well as the functionally-related RS-latch) the temporal displacement between the requests (or set and reset, respectively) will be the relevant property. For the Muller C-element we have to consider two cases, as outlined in Sec. 2.3, but ultimately again the displacement of input edges is the decisive quantity. So we can again apply Eq. (3), but this time just for expressing the delay as a function of $\Delta T$, 
and we can apply Eq. (5) to retain the same characteristic parameters. This yields a delay of

$$
\delta(\Delta T)=t_{\mathrm{res}}(\Delta T)=\tau \cdot \ln \left(\frac{T_{0}}{\Delta T}\right) .
$$

Figure 5 shows an example plot for the RS-latch, according to a SPICE simulation of an actual circuit implementation (the one given in Sec. 2.3). This curve is, of course, highly nonlinear and hence not very convenient. However, Eq. (6) suggests a linear graph when plotting $\delta$ over $\log (\Delta T)$. Figure 8 shows the transformed plot. Indeed we observe a largely linear curve now, however with a bend towards higher values of $\Delta T$, where the model assumptions (particularly the constant gain of the inverter) are no more valid. The slope at the left of the bend is determined by the element's resolution time constant $\tau$, while the horizontal part of the graph indicates a constant output delay for large enough values of $\Delta T$ - this is the nominal output delay in case of no metastability. In this curve $T_{0}$ can be found at the zero-crossing of the delay curve (i.e., the inflection point), as can be verified by setting Eq. (6) to zero.

This graph is much more useful for characterizing the behavior of a bistable element in elastic timing environments than the MTBU graph (in principle it can also be applied for the D-latch and the D-flip-flop if the actual delay is relevant). It displays the element's relevant property in a natural and intuitive way.

\subsection{Improving the metastability model}

While Veendrick's model from Eq. (2) describes the behavior in the exponential range of the delay graph extremely well, the area of constant delay outside the deep

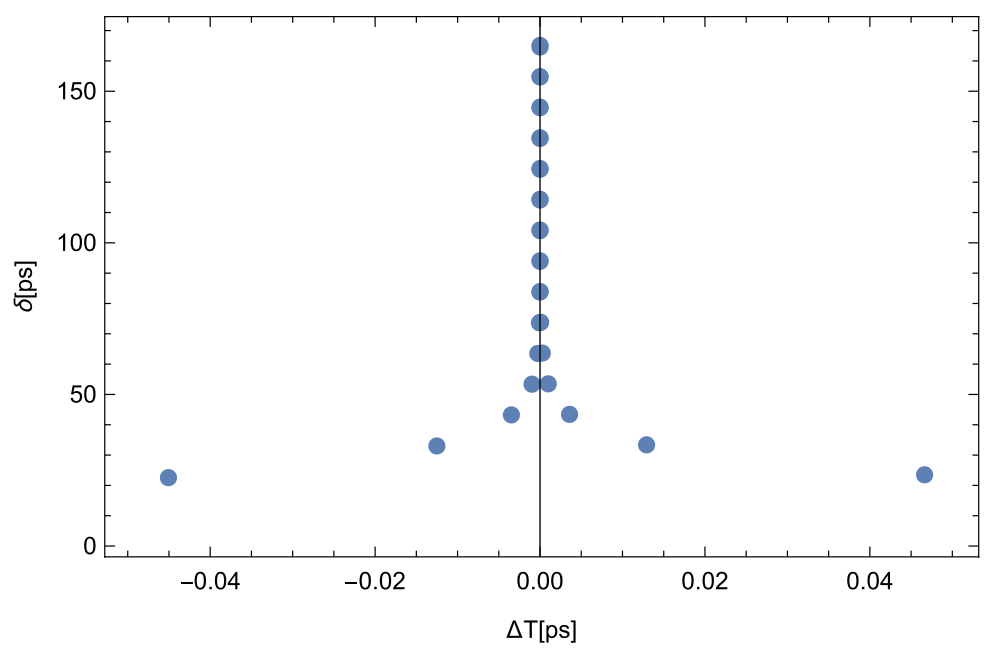

Fig. 5. The delay behavior of an RS-latch. 


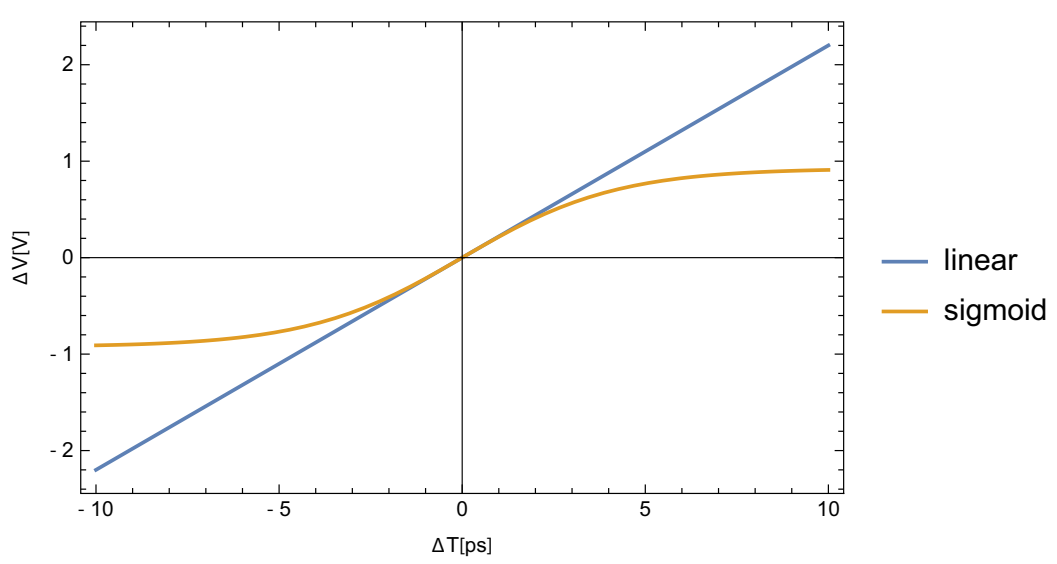

Fig. 6. Input overlap versus voltage difference model.

metastable region is not captured by this model at all. In this paper we therefore augment the model to be able to use a single equation for both areas. The key observation for our model refinement is that the relation between input overlap $(\Delta T)$ and initial offset voltage $\left(V_{\Delta}\right)$ is not a simple constant $\theta$, as modeled by Veendrick, which implies a linear characteristic, but saturates for larger input overlaps (see Fig. 6).

Of course the saturation could be considered by a horizontal line, thus turning the model into a piecewise function comprising two straight lines. This, however, has two disadvantages: (i) The piecewise function is more difficult to handle, e.g., when trying to find a suitable fitting to measured values, and (ii) as visible in Fig. 5, for example, the linear approximation becomes imprecise in the proximity of the intersection between the two straight lines, i.e., for very small resolution times. The latter is often unproblematic, as in practice operation with small resolution time is usually avoided, but for aggressive timing and when more closely investigating the handover between stages — such as between master and slave in the flip-flop, or between stages of a synchronizer - a precise model for that area becomes necessary.

While we had already pursued the approach of deriving an analytic function for the whole range earlier, the resulting model was quite complex and its fitting process therefore tedious. ${ }^{10}$ The model we present here shall remedy this shortcoming. We choose to use a sigmoid function to capture the $V_{\Delta}(\Delta T)$ characteristic. The equation is governed by two parameters, namely the maximum slope of the sigmoid $\left(\frac{\theta}{v_{s}}\right)$ (in the zero-crossing) and its height $\left(v_{s}\right)$ :

$$
V_{\Delta}(\Delta T)=v_{s}\left(\frac{1}{1+e^{-\Delta T \theta}}-\frac{1}{2}\right) .
$$




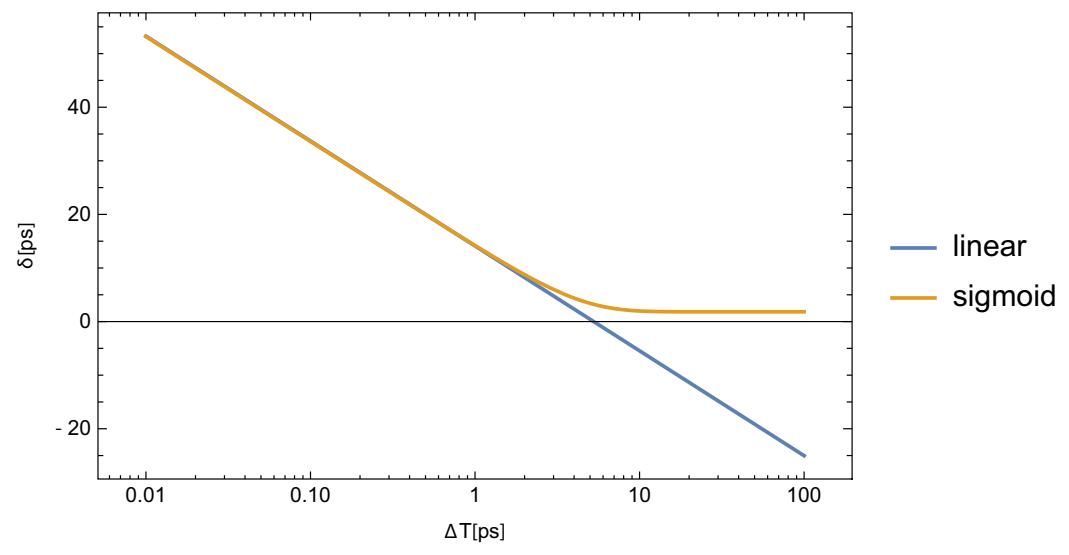

Fig. 7. The delay models comparison.

Following the same steps as Veendrick, we can determine the time-dependent output voltage $V_{\text {out }}$ by substituting $V_{\Delta}$ in Eq. (1) by Eq. (7) (Ref. 2):

$$
V_{\text {out }}(t, \Delta T)=v_{s}\left(\frac{1}{1+e^{-\Delta T \theta}}-\frac{1}{2}\right) e^{\frac{t}{\tau}} .
$$

Solving for $t$, the time for metastability resolution $t_{\text {res }}$ can be calculated as

$$
t_{\mathrm{res}}\left(\Delta T, V_{\text {out }}\right)=\tau \ln \left(\frac{2\left(e^{\Delta T \theta}+1\right) V_{\text {out }}}{\left(e^{\Delta T \theta}-1\right) v_{s}}\right) .
$$

Setting the output voltage $V_{\text {out }}$ to the threshold voltage $V_{\text {th }}$, the metastability delay can be expressed as

$$
\delta(\Delta T)=\tau \ln \left(\frac{2\left(e^{\Delta T \theta}+1\right) V_{\mathrm{th}}}{\left(e^{\Delta T \theta}-1\right) v_{s}}\right),
$$

which can be more concisely written as (with $\left.r_{v}=\frac{V_{\text {th }}}{v_{s}}\right)$

$$
\delta(\Delta T)=\tau \ln \left(2 r_{v} \operatorname{coth} \frac{\Delta T \theta}{2}\right) .
$$

In contrast to the classic result (Eq. (6)), for rising $\Delta T$, the logarithmic behavior changes to a constant one (see Fig. 7 for an example).

\subsection{Determination of the relevant parameters}

The actual delay graph can be experimentally obtained by measuring $\delta$ as a function of $\Delta T$. This can in principle be done by the conventional means of delay measurement, like an oscilloscope or a counter. Interestingly, the late transition detector circuits usually employed for obtaining the MTBU curve can be beneficially used 
here as well. ${ }^{11}$ However, the interpretation of the readouts then differs in detail from the one applied for MTBU prediction: As already outlined, here we are not concerned with discriminating "proper" reaction times from "upsets"; we are just measuring the delay without a need for interpreting it. It is important to note here that the measurement principle applied in the detection circuit does not allow an absolute calibration of the delay, i.e., just the difference between delays for different settings of $\Delta T$ can be determined. While this is still sufficient to calculate the slope of the delay curve, hence $\tau$, we cannot reliably determine the appropriate absolute position of the curve in the graph. This is not a problem of our approach alone, but a notorious, unsolved problem in metastability measurement.

In Sec. 4.2 we will further investigate how well our approximation by a sigmoid function reflects the true shape of the delay curve.

\subsection{Estimating performance penalty}

Based on the delay function we can express the performance penalty incurred by metastability resolution time in an asynchronous setting. In essence, some $\delta$ adds to the regular round time $t_{R}$ (full handshake cycle) whenever the sequential element gets metastable. Subsequently, we want to calculate the average delay $\overline{\Delta_{m}}=$ $\overline{\Delta_{m_{l}}}+\overline{\Delta_{m_{u}}}$ per round that is caused by those incidental metastability resolution delays:

$$
\overline{t_{R}}=t_{R}+\overline{\Delta_{m_{l}}}+\overline{\Delta_{m_{u}}}
$$

Here $\overline{\Delta_{m}}$ is given by the access delay due to metastability (with $\overline{\Delta_{m_{l}}}$ being the excess delay for all cases where the overlap is not sufficiently large to change the output ${ }^{\mathrm{b}}$ and $\overline{\Delta_{m_{u}}}$ for cases where it is).

To calculate the excess delay, we subtract the nominal output delay from Eq. (11). The nominal output delay is defined as the delay for an infinite large signal overlap:

$$
\lim _{\Delta T \rightarrow \infty} \delta_{\text {res }}=\lim _{\Delta T \rightarrow \infty}\left(\tau \ln \left(2 r_{v} \operatorname{coth} \frac{\Delta T \theta}{2}\right)\right)=\tau \ln \left(2 r_{v}\right) .
$$

We integrate the resulting equation from the balance point to the overlap $\left(\Delta_{R}\right)$ corresponding to the nominal round duration $\left(t_{R}\right)$ and divide the result by the nominal round duration (assuming $\Delta T$ to be equally distributed within the round duration and $\overline{\Delta_{m}} \ll t_{R}$ so it can be neglected in the denominator). For $\overline{\Delta_{m_{l}}}$ we go to

\footnotetext{
b Even if in the end the sequential element decides to retain the state it had prior to becoming metastable, it is in a metastable state for some time, which may become observable as a glitch. It depends on the design whether and how this is handled. Here we provide all required parameters for doing the calculations appropriate to the given problem.
} 
the left of the balance point, while for $\overline{\Delta_{m_{u}}}$ we go to the right:

$$
\begin{aligned}
\overline{\Delta_{m_{l / u}}} & \approx \frac{\int_{0}^{\Delta_{R_{l / u}}}\left(\tau_{l / u} \ln \left(2 r_{v_{l / u}} \operatorname{coth} \frac{\Delta T \theta_{l / u}}{2}\right)-\tau_{l / u} \ln \left(2 r_{v_{l / u}}\right)\right) d \Delta T}{t_{R}} \\
& =\frac{\int_{0}^{\Delta_{R_{l / u}}}\left(\tau_{l / u} \ln \operatorname{coth} \frac{\Delta T \theta_{l / u}}{2}\right) d \Delta T}{t_{R}} .
\end{aligned}
$$

As the delay penalty near $\Delta_{R_{l / u}}$ is almost zero for typical round durations, we can replace the upper integration limit with $\infty$ with only a marginal error in the result (thus overestimating the penalty). Therefore $\overline{\Delta_{m_{l / u}}}$ evaluates to

$$
\overline{\Delta_{m_{l / u}}} \approx \frac{\int_{0}^{\infty}\left(\tau_{l / u} \ln \operatorname{coth} \frac{\Delta T \theta_{l / u}}{2}\right) d \Delta T}{t_{R}}=\frac{\pi^{2} \tau_{l / u}}{4 \theta_{l / u} t_{R}} .
$$

\section{Example Studies}

\subsection{Overall behavior}

We have performed SPICE simulations (pre-layout, parasitics are not considered) of the elements presented in Secs. 2.2 and 2.3 in an industrial $65 \mathrm{~nm}$ technology and will plot the delay in the proposed way. This shall allow us to conveniently compare the properties of these circuits. The size of the PMOS transistor was chosen twice the size of the NMOS transistor. It was kept constant for all simulated elements. Only the feedback inverter in the weak-feedback MCE was chosen half the size of the others.
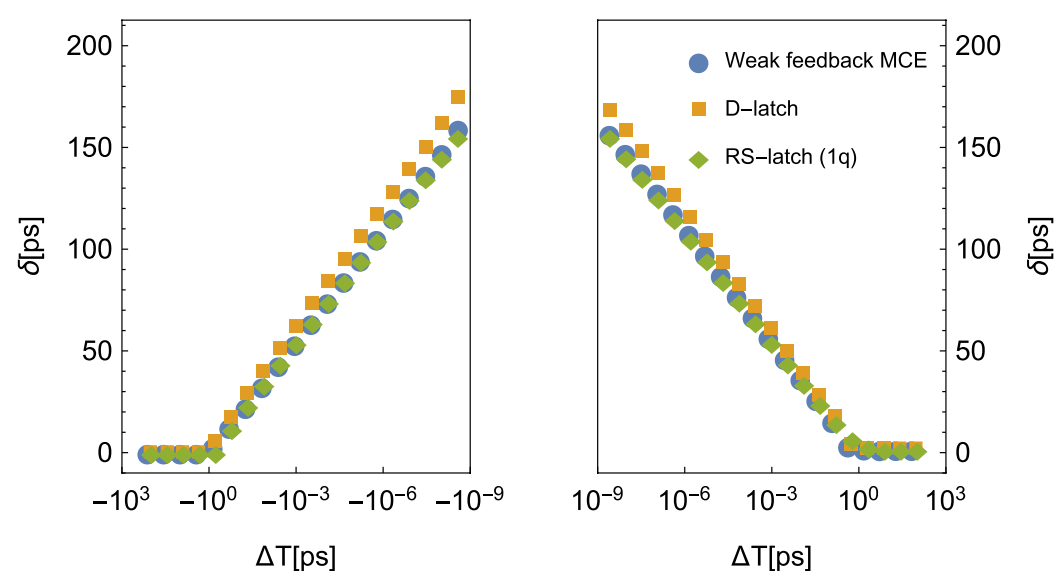

Fig. 8. Metastabilities comparison of a Muller C-element, a D-latch and an RS-latch. 

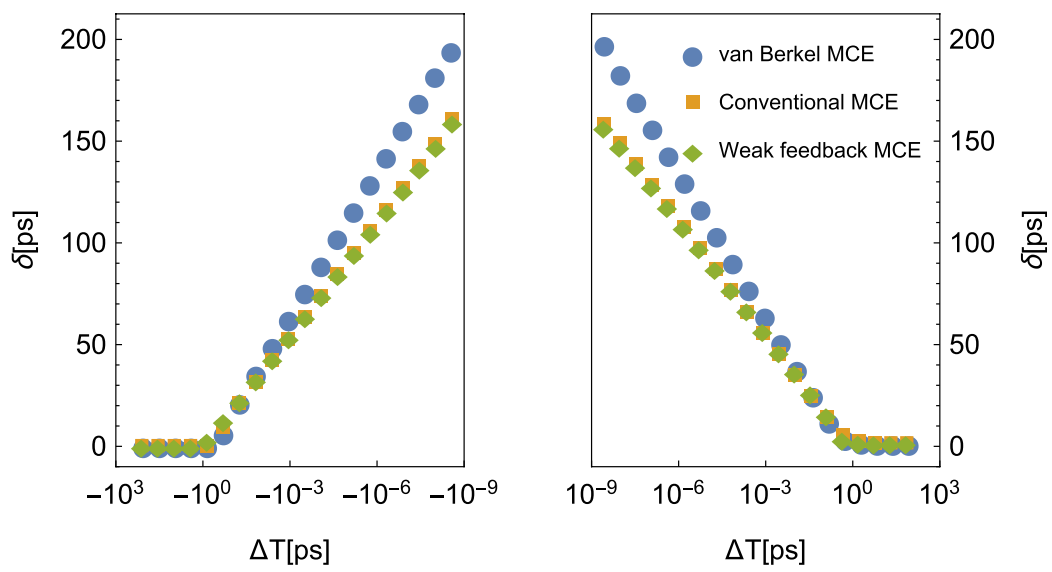

Fig. 9. Metastabilities comparison of the different Muller C-element implementations.

Figure 8 shows the weak-feedback MCE, the D-latch and the RS-latch. The left part of the figure visualizes input overlaps shorter than the balance point, while the right side visualizes the ones larger. It can be seen that our postulated logarithmic dependence (straight line in a semilogarithmic scale) between the distance of the input overlap from its balance point and the output delay holds in all three cases. Further the deviation of this trend for large distances is clearly visible (the horizontal line on the far corners of the subgraphs). All three elements have different metastability resolution behaviors. The element with the fastest recovery is the weak-feedback MCE, while the D-latch is the slowest. The differences are due to the different circuit
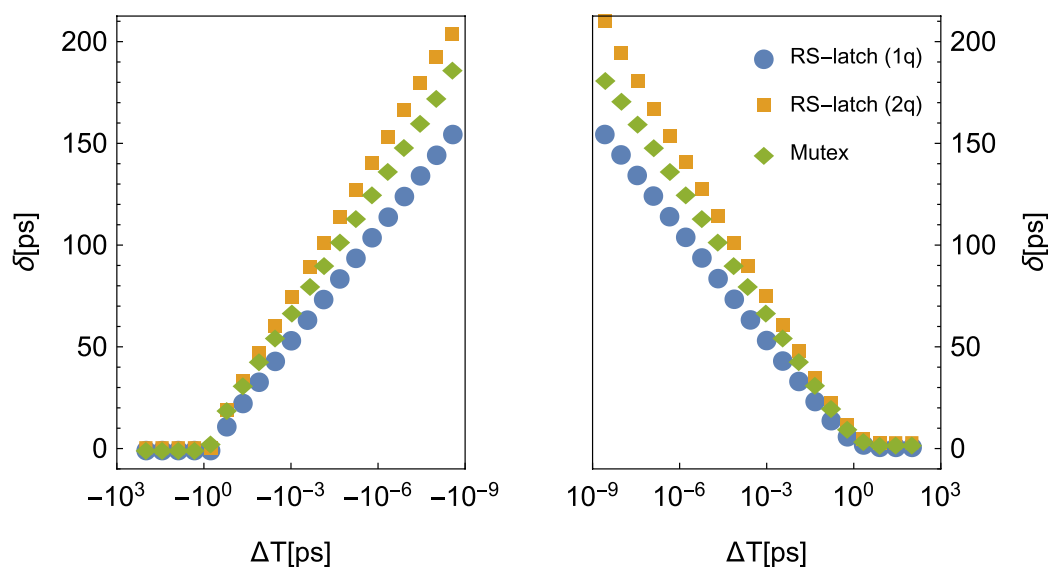

Fig. 10. Metastabilities comparison of an RS-latch and a mutex. 

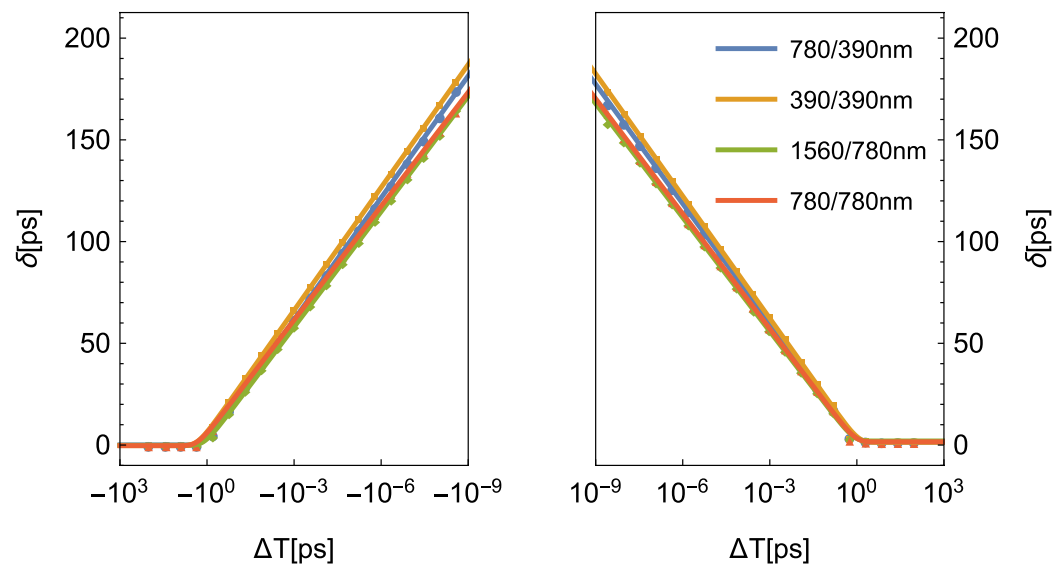

Fig. 11. Metastabilities comparison of different D-latch sizings.

structures of the elements, as capacitive loading, for example, is known to have a significant impact on metastability resolution behavior.

Additional results for the other MCE implementations are plotted in Fig. 9. It can be seen that, again, the different circuit structures lead to quite different slopes of the graphs but the overall shape stays unchanged and our postulated behavior is valid in these cases.

Finally, we have compared the mutex element with the RS-latch (Fig. 10). As their sizings are the same, one would assume nearly-identical resolution behavior. The results, however, show that the mutex resolves metastability worse than the RSlatch. The reason for the different behavior is the second output of the mutex
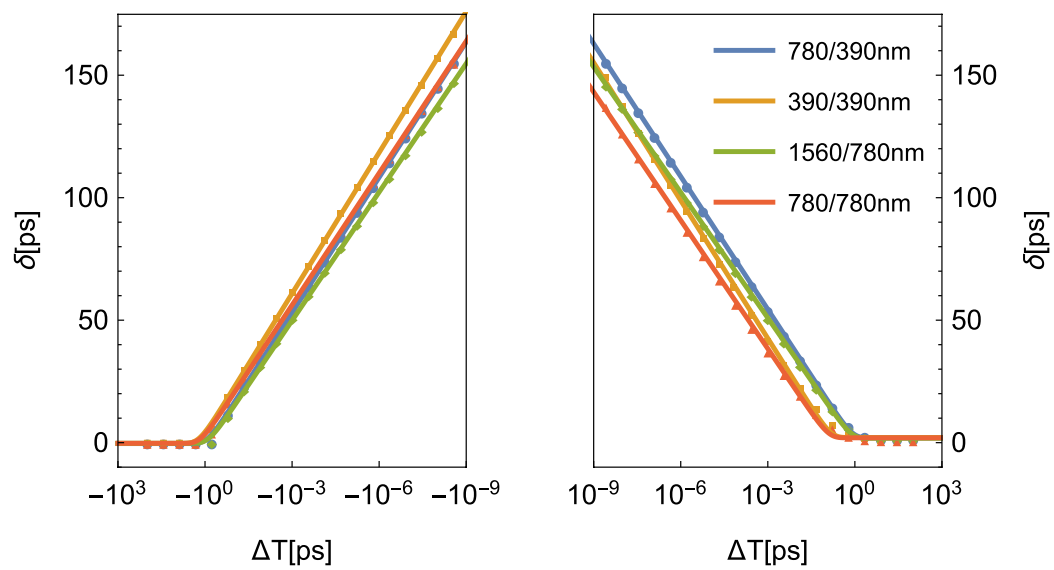

Fig. 12. Metastabilities comparison of different RS-latch sizings (one output). 

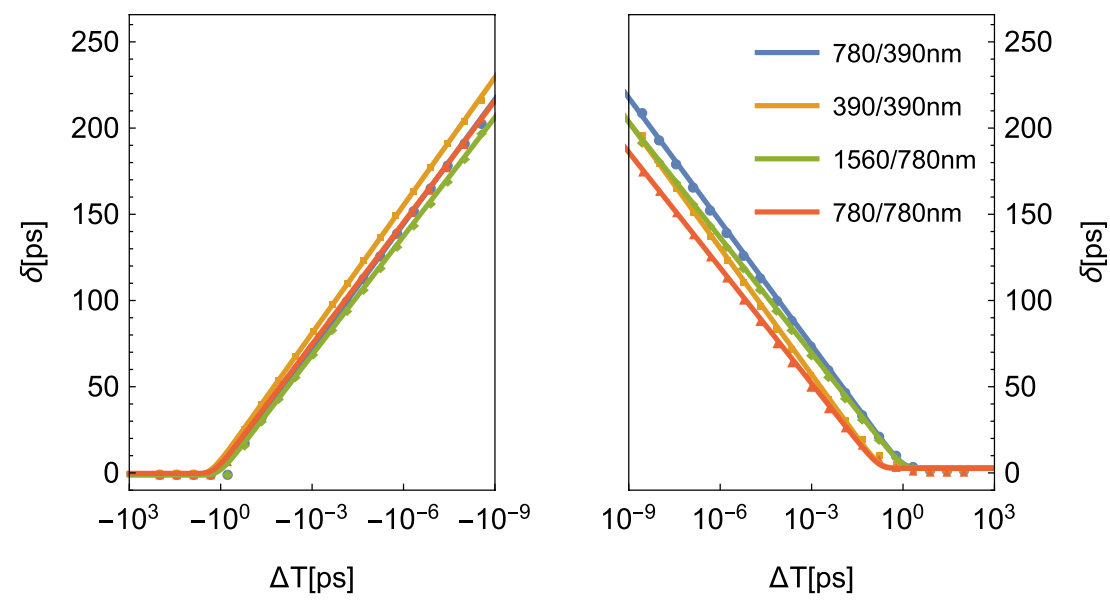

Fig. 13. Metastabilities comparison of different RS-latch sizings (two outputs).

element. It adds load to the storage loop and therefore worsens the resolution behavior. To illustrate this further, we have added a second, inverted output $\left(Q_{b}\right)$ to the RS-latch (green curve) by connecting an inverter to the noninverting loop node $(z)$. The result was an even worse resolution behavior as in the case of mutex.

\subsection{Model fitting}

To demonstrate the viability of our newly derived mode, we performed a curve fitting of our proposed function with the transistor-level simulation results from the real
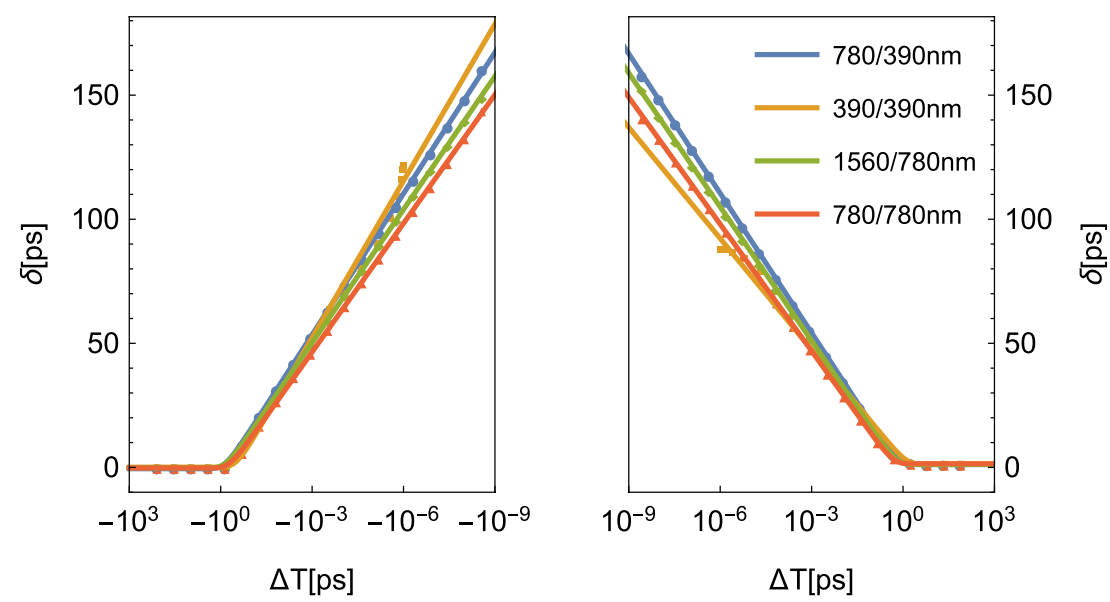

Fig. 14. Metastabilities comparison of different conventional C-element sizings. 

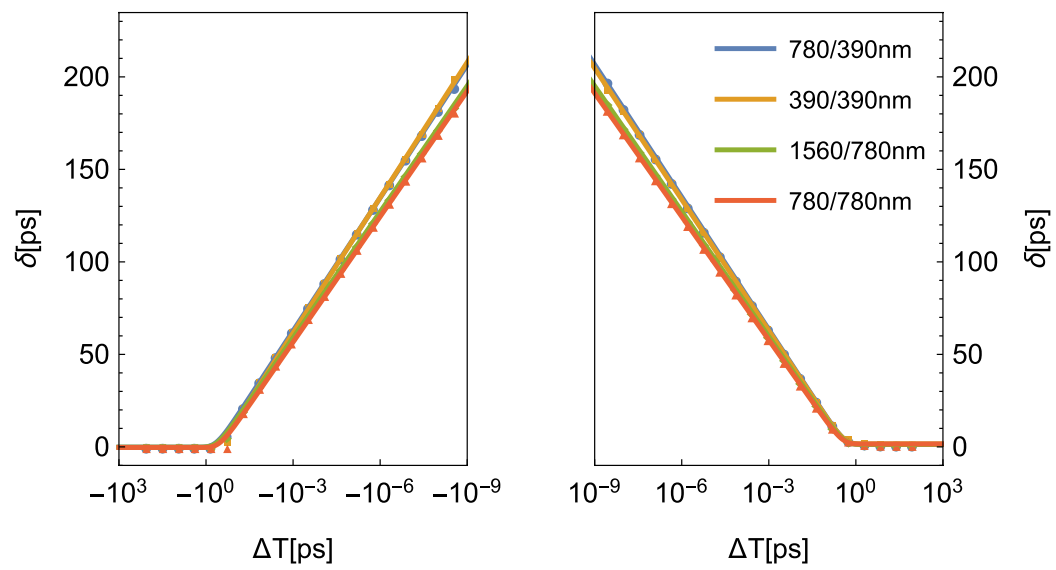

Fig. 15. Metastabilities comparison of different van Berkel C-element sizings.

circuits described above. We varied the widths of the $p$ - and $n$-channel MOSFETs in the simulations. The results are depicted in Figs. 11-17 with the following color coding:

- blue: $W(p-\mathrm{MOS})=780 \mathrm{~nm}, W(n-\mathrm{MOS})=390 \mathrm{~nm}$;

- yellow: $W(p-\mathrm{MOS})=390 \mathrm{~nm}, W(n-\mathrm{MOS})=390 \mathrm{~nm}$;

- green: $W(p$-MOS $)=1,560 \mathrm{~nm}, W(n-\mathrm{MOS})=780 \mathrm{~nm}$;

- orange: $W(p-\mathrm{MOS})=780 \mathrm{~nm}, W(n-\mathrm{MOS})=780 \mathrm{~nm}$.

The figures show that the fitting of the model works very well and the simulation results are closely tracked. As the rounding between the constant and logarithmic behaviors is solely determined by the chosen sigmoid equation, the fitting process
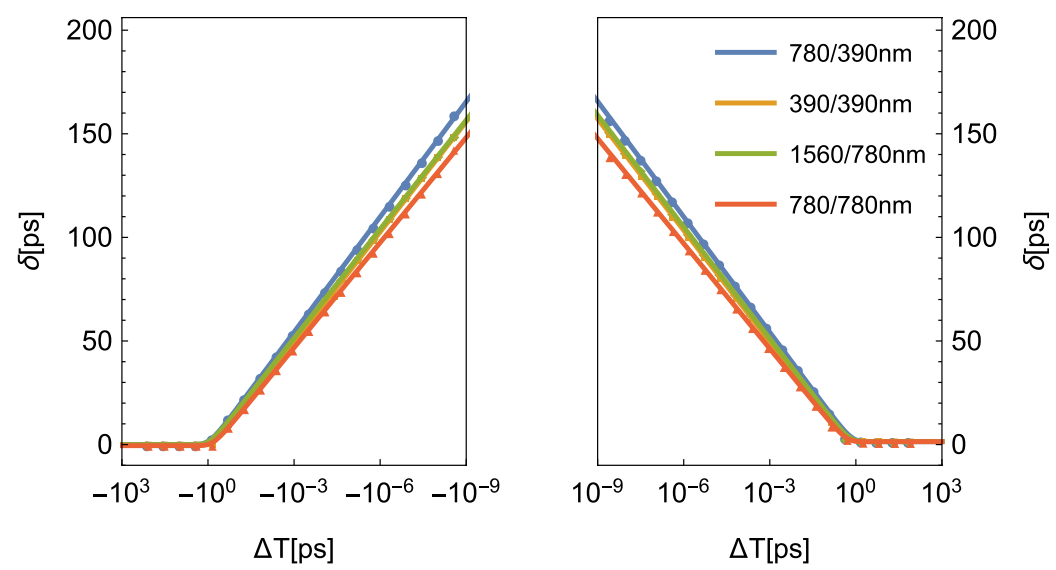

Fig. 16. Metastabilities comparison of different weak-feedback C-element sizings. 

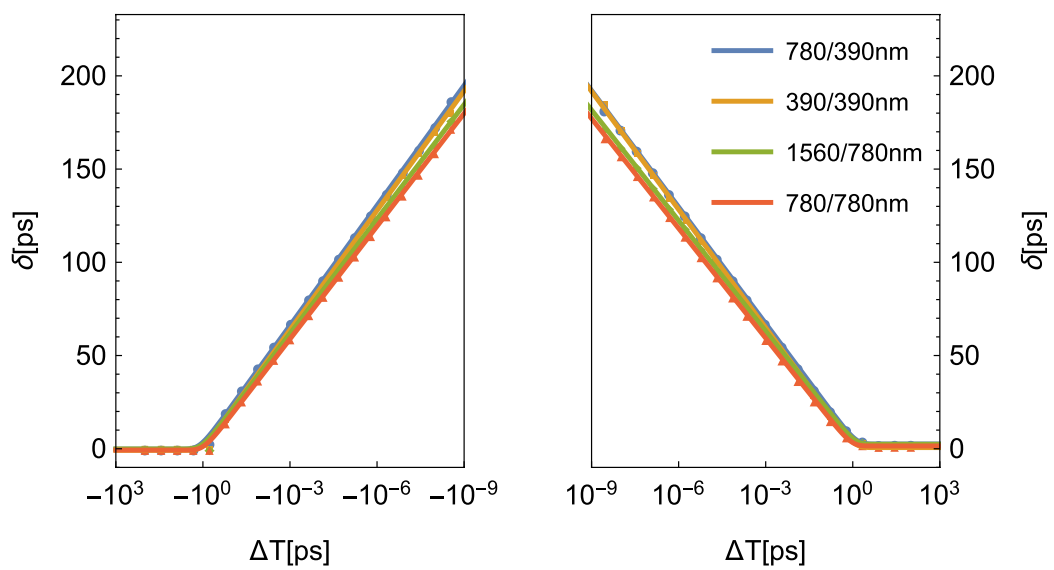

Fig. 17. Metastabilities comparison of different mutex sizings.

Table 1. Fitted parameters.

\begin{tabular}{|c|c|c|c|c|c|c|c|c|}
\hline & $p$ & $n$ & $\tau_{l}$ & $r_{v_{l}}$ & $\theta_{l}$ & $\tau_{u}$ & $r_{v_{u}}$ & $\theta_{u}$ \\
\hline Circuit & $\mathrm{nm}$ & $\mathrm{nm}$ & ps & 1 & $1 / \mathrm{ps}$ & ps & 1 & $1 / \mathrm{ps}$ \\
\hline D-latch & 780 & 390 & 8.67002 & 0.503714 & 1.64715 & 8.49889 & 0.590586 & 2.045 \\
\hline D-latch & 390 & 390 & 8.744 & 0.483787 & 0.995269 & 8.70292 & 0.584316 & 1.83303 \\
\hline D-latch & 1,560 & 780 & 8.20711 & 0.493161 & 1.67261 & 8.09951 & 0.639899 & 2.57094 \\
\hline D-latch & 780 & 780 & 8.10309 & 0.481938 & 0.983587 & 8.18717 & 0.608241 & 2.27605 \\
\hline RS-latch $(1 q)$ & 780 & 390 & 7.9979 & 0.477338 & 2.50659 & 7.84947 & 0.641383 & 2.50237 \\
\hline RS-latch $(1 q)$ & 390 & 390 & 8.33354 & 0.481118 & 1.29488 & 8.14953 & 0.646392 & 13.9393 \\
\hline RS-latch $(1 q)$ & 1,560 & 780 & 7.59268 & 0.484046 & 2.72266 & 7.41706 & 0.637781 & 2.66377 \\
\hline RS-latch $(1 q)$ & 780 & 780 & 7.79759 & 0.483722 & 1.44093 & 7.58224 & 0.649529 & 16.4801 \\
\hline RS-latch $(2 q)$ & 780 & 390 & 10.4701 & 0.481118 & 1.96616 & 10.3809 & 0.668362 & 2.11944 \\
\hline RS-latch $(2 q)$ & 390 & 390 & 10.7404 & 0.459924 & 0.974465 & 10.6021 & 0.659279 & 12.0402 \\
\hline RS-latch $(2 q)$ & 1,560 & 780 & 9.97366 & 0.439462 & 1.84173 & 9.69331 & 0.650878 & 2.00337 \\
\hline RS-latch $(2 q)$ & 780 & 780 & 10.221 & 0.487135 & 1.31232 & 9.70877 & 0.656208 & 12.5559 \\
\hline van Berkel MCE & 780 & 390 & 10.3986 & 0.494204 & 4.71718 & 10.3668 & 0.571912 & 5.00354 \\
\hline van Berkel MCE & 390 & 390 & 10.5874 & 0.488669 & 5.78676 & 10.2659 & 0.586869 & 5.17675 \\
\hline van Berkel MCE & 1,560 & 780 & 9.85484 & 0.491976 & 4.95529 & 9.80643 & 0.572002 & 5.24533 \\
\hline van Berkel MCE & 780 & 780 & 9.82014 & 0.484056 & 6.06008 & 9.65334 & 0.588453 & 5.90638 \\
\hline Conventional MCE & 780 & 390 & 8.23209 & 0.46922 & 2.83421 & 8.1409 & 0.57534 & 2.94637 \\
\hline Conventional MCE & 390 & 390 & 9.15324 & 0.504792 & 6.72682 & 6.47667 & 0.607199 & 1.58133 \\
\hline Conventional MCE & 1,560 & 780 & 7.77423 & 0.487332 & 3.047 & 7.80636 & 0.582941 & 3.36629 \\
\hline Conventional MCE & 780 & 780 & 7.50701 & 0.484089 & 3.95926 & 7.402 & 0.611076 & 4.33237 \\
\hline Weak-FB MCE & 780 & 390 & 8.04608 & 0.503122 & 2.35318 & 8.06606 & 0.59481 & 2.87307 \\
\hline Weak-FB MCE & 390 & 390 & 7.70734 & 0.488648 & 3.21234 & 7.77766 & 0.607005 & 4.06951 \\
\hline Weak-FB MCE & 1,560 & 780 & 7.62231 & 0.493569 & 2.34911 & 7.80466 & 0.604212 & 3.45181 \\
\hline Weak-FB MCE & 780 & 780 & 7.34437 & 0.465758 & 3.20212 & 7.34194 & 0.604635 & 4.40547 \\
\hline Mutex & 780 & 390 & 9.32073 & 0.489155 & 1.62504 & 9.04851 & 0.663649 & 1.58893 \\
\hline Mutex & 390 & 390 & 9.26995 & 0.456657 & 1.87812 & 9.17306 & 0.535508 & 1.80047 \\
\hline Mutex & 1,560 & 780 & 8.89169 & 0.494886 & 1.90451 & 8.5832 & 0.662886 & 1.70508 \\
\hline Mutex & 780 & 780 & 8.7581 & 0.461277 & 2.22488 & 8.52363 & 0.596592 & 2.26147 \\
\hline
\end{tabular}


Table 2. Calculated $\overline{\Delta_{m}}$ values.

\begin{tabular}{|c|c|c|c|c|}
\hline & $p$ & $n$ & $\overline{\Delta_{m_{l}}} \cdot t_{R}$ & $\overline{\Delta_{m_{u}}} \cdot t_{R}$ \\
\hline Circuit & $\mathrm{nm}$ & $\mathrm{nm}$ & $p s^{2}$ & $p s^{2}$ \\
\hline D-latch & 780 & 390 & 12.9875 & 10.2544 \\
\hline D-latch & 390 & 390 & 21.6775 & 11.7148 \\
\hline D-latch & 1,560 & 780 & 12.107 & 7.77333 \\
\hline D-latch & 780 & 780 & 20.3272 & 8.87549 \\
\hline RS-latch $(1 q)$ & 780 & 390 & 7.87285 & 7.73977 \\
\hline RS-latch $(1 q)$ & 390 & 390 & 15.8796 & 1.44255 \\
\hline RS-latch $(1 q)$ & 1,560 & 780 & 6.88084 & 6.87029 \\
\hline RS-latch $(1 q)$ & 780 & 780 & 13.3523 & 1.13521 \\
\hline RS-latch $(2 q)$ & 780 & 390 & 13.1392 & 12.0852 \\
\hline RS-latch $(2 q)$ & 390 & 390 & 27.1953 & 2.17269 \\
\hline RS-latch $(2 q)$ & 1,560 & 780 & 13.3619 & 11.9385 \\
\hline RS-latch $(2 q)$ & 780 & 780 & 19.2172 & 1.9079 \\
\hline van Berkel MCE & 780 & 390 & 5.43916 & 5.11221 \\
\hline van Berkel MCE & 390 & 390 & 4.51433 & 4.89305 \\
\hline van Berkel MCE & 1,560 & 780 & 4.90704 & 4.61294 \\
\hline van Berkel MCE & 780 & 780 & 3.99833 & 4.0327 \\
\hline Conventional MCE & 780 & 390 & 7.16669 & 6.8175 \\
\hline Conventional MCE & 390 & 390 & 3.35741 & 10.1057 \\
\hline Conventional MCE & 1,560 & 780 & 6.29543 & 5.72185 \\
\hline Conventional MCE & 780 & 780 & 4.67835 & 4.21564 \\
\hline Weak-feedback MCE & 780 & 390 & 8.43664 & 6.92716 \\
\hline Weak-feedback MCE & 390 & 390 & 5.92002 & 4.71571 \\
\hline Weak-feedback MCE & 1,560 & 780 & 8.00614 & 5.57887 \\
\hline Weak-feedback MCE & 780 & 780 & 5.65923 & 4.11205 \\
\hline Mutex & 780 & 390 & 14.1523 & 14.0512 \\
\hline Mutex & 390 & 390 & 12.1785 & 12.571 \\
\hline Mutex & 1,560 & 780 & 11.5197 & 12.4206 \\
\hline Mutex & 780 & 780 & 9.71279 & 9.29979 \\
\hline
\end{tabular}

cannot fully fit the curves in this area but only the selection of another sigmoid representation could. However, the resulting deviation between the simulation data and the fitted model is small. The fitted model parameters for our example studies can be found in Table 1 , while the calculated $\overline{\Delta_{m}}$ values are summarized in Table 2 .

It can be observed that the $\overline{\Delta_{m}}$ values vary significantly with the implementation, which is mainly due to the variation of $\theta$. Generally, however, the performance penalty is very low: For a reasonable choice of $t_{R}=10 \mathrm{~ns}$ it is in the range of $1 \mathrm{ppm}$.

Although we covered a lot of different cases, the analysis in this paper was limited to $65 \mathrm{~nm}$ technology and "typical case" simulation. The impact of technology scaling on metastability parameters has already been extensively studied, and, although the conclusions differ in detail, a strong dependence of the parameters on technology was 
reported in all cases. ${ }^{12-14}$ Similarly, a strong dependence of temperature and voltage on the metastability parameters has been reported. ${ }^{15}$ These effects, however, are somewhat orthogonal to the line of arguments that we followed in this paper: Changing parameters sets (for the same underlying type of behavior) can be easily accommodated in our model without changing its fundamental structure or the key observations that we reported above.

\section{Conclusion}

Based on the observation that MTBU is not an appropriate means of characterizing the metastable behavior of sequential elements used in environments with elastic timing, we have presented the delay graph as a logical, intuitive alternative. For a reasonably high degree of metastability, this graph becomes a straight line which allows direct observation of the relevant parameters $\tau$ and $T_{0}$. However, with metastability becoming weaker, the graph bends towards a constant value. We have included this effect in our model by using a sigmoid function. We have shown how to fit the model parameters to actual measurements or results from transistor-level simulations. A very good fitting could be achieved for all popular sequential elements and different implementations thereof. In addition, our proposed presentation as a delay graph allows a very intuitive comparison of the performances of different alternatives.

In contrast to measurement and simulation, our model offers the significant benefit of having an analytic function for the complete delay graph available. By appropriately integrating this function we could obtain an equation for the average performance penalty through occasional metastability delays. It confirms the very low penalty that is commonly assumed but has not been calculated in that level of detail so far.

\section{Acknowledgment}

This research was supported by the SIC Project (Grant No. P26436-N30) of the Austrian Science Fund (FWF).

\section{References}

1. L. R. Marino, General theory of metastable operation, IEEE Trans. Comput. C-30 (1981) $107-115$.

2. H. J. Veendrick, The behaviour of flip-flops used as synchronizers and prediction of their failure rate, IEEE J. Solid-State Circuits 15 (1980) 169-176.

3. M. S. Haydt, S. Mourad, W. Terry and J. Terry, A new model for metastability, Proc. 9th Int. Conf. Electronics, Circuits and Systems, Vol. 1, Dubrovnik, Croatia (2002), pp. 413-416. 
4. T. Polzer and A. Steininger, Enhanced metastability characterization based on AC analysis, Proc. 2015 Euromicro Conf. Digital System Design, Funchal, Madeira, Portugal (2015), pp. 722-729.

5. T. Polzer and A. Steininger, Metastability characterization for Muller C-elements, Proc. 2013 23rd Int. Workshop Power and Timing Modeling, Optimization and Simulation (PATMOS), Karlsruhe (2013), pp. 164-171.

6. J. Sparsø and S. Furber, Principles of Asynchronous Circuit Design - A Systems Perspective (Springer Science and Business Media, 2001).

7. M. Shams, J. C. Ebergen and M. I. Elmasry, A comparison of CMOS implementations of an asynchronous circuits primitive: The C-element, Proc. Int. Symp. Low Power Electronics and Design, Piscataway, NJ, USA (1996), pp. 93-96.

8. T. Polzer, A digital metastability model for VLSI circuits, Ph.D. thesis, TU Wien, Vienna (2013).

9. C. L. Seitz, System timing, Introduction to VLSI Systems, eds. C. Mead and L. Conway (Addison-Wesley, 1979).

10. T. Polzer and A. Steininger, Digital late-transition metastability simulation model, 2013 Euromicro Conf. Digital System Design (DSD), Santander (2013), pp. 121-128.

11. T. Polzer and A. Steininger, An approach for efficient metastability characterization of FPGAs through the designer, Proc. 19th IEEE Int. Symp. Asynchronous Circuits and Systems (ASYNC), Santa Monica (2013), pp. 174-182.

12. M. S. Baghini and M. P. Desai, Impact of technology scaling on metastability performance of CMOS synchronizing latches, Proc. ASP-DAC/VLSI Design 2002: 7th Asia and South Pacific Design Automation Conf. and 15th Int. Conf. VLSI Design (IEEE, 2002), pp. 317-322.

13. S. Beer, R. Ginosar, M. Priel, R. Dobkin and A. Kolodny, The devolution of synchronizers, Proc. 2010 IEEE Symp. Asynchronous Circuits and Systems, Grenoble, France (2010), pp. 94-103.

14. S. Yang, I. W. Jones and M. R. Greenstreet, Synchronizer performance in deep sub-micron technology, Proc. 2011 17th IEEE Int. Symp. Asynchronous Circuits and Systems, Ithaca, New York (2011), pp. 33-42.

15. S. Beer and R. Ginosar, A model for supply voltage and temperature variation effects on synchronizer performance, IEEE Trans. Very Large Scale Integr. (VLSI) Syst. 23 (2015) $2461-2472$. 